\title{
Nucleon form factors induced by isovector and isoscalar axial-vector currents in QCD
}

\author{
T. M. Aliev *†, M. Savcl $\ddagger$ \\ Physics Department, Middle East Technical University, 06531 Ankara, Turkey
}

\begin{abstract}
Using the most general form of the baryon current, nucleon form factors, induced by isovector and isoscalar axial-vector currents, are studied in the framework of light cone QCD sum rule approach. Comparison of our results on form factors with the existing results and lattice calculations are presented.
\end{abstract}

PACS number(s): 11.55.Hx, 13.40.Em, 14.20.Jn

*e-mail: taliev@metu.edu.tr

${ }^{\dagger}$ permanent address:Institute of Physics,Baku,Azerbaijan

†e-mail: savci@metu.edu.tr 


\section{Introduction}

It is well known that the structure of the nucleon is parametrized in terms of form factors. The electromagnetic form factors of nucleon are measured in a wide range of momentum transfer squared $q^{2}$ (see for example [1] and the references therein). But, in contrast to the electromagnetic case, the form factors $G_{A}\left(q^{2}\right), G_{P}\left(q^{2}\right)$ and $G_{T}\left(q^{2}\right)$ due to isovector axial-vector current are not known. The nucleon matrix elements of axial-vector currents at $q^{2}=0$ are determined by the axial-vector coupling constants such as $g_{A}$ (isovector), $g_{A}^{8}$ (octet), $g_{A}^{S}$ (isoscalar) and $g_{A}^{0}$ (flavor singlet). Among these only nucleon isovector coupling constant $g_{A}$ is well known which is measured from the neutron $\beta$-decay. Knowledge of any three of these coupling constants determines the quark spin content of the nucleon. For this reason study of these constants receives great interest.

Using the Lorentz covariance, the matrix element of the isovector axial-vector current between initial and final states is parametrized as

$$
\left\langle N\left(p^{\prime}\right)\left|A_{\mu}\right| N(p)\right\rangle=\bar{u}\left(p^{\prime}\right)\left[\gamma_{\mu} \gamma_{5} G_{A}\left(q^{2}\right)+\frac{q_{\mu}}{2 m_{N}} \gamma_{5} G_{P}\left(q^{2}\right)+i \sigma_{\mu \nu} \frac{q^{\nu}}{2 m_{N}} \gamma_{5} G_{T}\left(q^{2}\right)\right] u(p)
$$

where $A_{\mu}=\bar{u} \gamma_{\mu} \gamma_{5} u-\bar{d} \gamma_{\mu} \gamma_{5} d, q=p-p^{\prime}, m_{N}$ is the nucleon mass, and $G_{A}, G_{P}$ and $G_{T}$ are the axial, induced pseudoscalar and induced tensorial form factors, respectively, induced by the isovector and axial-vector currents. The matrix element of isoscalar axial-vector current $A_{\mu}^{S}=\bar{u} \gamma_{\mu} \gamma_{5} u+\bar{d} \gamma_{\mu} \gamma_{5} d$ between nucleon states is determined similar to (11) with the following replacements: $G_{A} \rightarrow G_{A}^{S}, G_{P} \rightarrow G_{P}^{S}$ and $G_{T} \rightarrow G_{T}^{S}$ (here the superscript $S$ means isoscalar). The form factors $G_{T}$ and $G_{T}^{S}$ both vanish as a result of the exact isospin symmetry and $\mathrm{G}$-parity invariance of the strong interaction.

The aim of the present work is the calculation of the form factors $G_{A}\left(G_{A}^{S}\right)$ and $G_{P}\left(G_{P}^{S}\right)$ in the framework of light cone QCD sum rules (LCSR) [3, 4] approach, using the most general form of nucleon interpolating current. LCSR is based on the operator product expansion over twist of the operators near the light cone. This method combines the standard sum rules technique [5] with the parton distribution amplitudes describing the hard exclusive processes. This method is widely applied to the problems in the meson sector (see [4]). Lately, the electromagnetic form factors of nucleon [6], the scalar form factor of nucleon [7] and the weak $\Lambda_{b} \rightarrow p \ell \nu$ [8] are investigated in the baryonic sector in the framework of this method. Note that higher twist amplitudes of the nucleon are calculated in [9].

The outline of this work is as follows. In section 2 the basic ingredients for calculating the form factors due to axial-vector current are introduced and sum rules for the form factors are constructed. In section 3 we present our numerical results together with the concluding remarks.

\section{Sum rules for the form factors of the nucleon due to isovector and isoscalar currents}

In this section, we construct sum rules for the form factors of the nucleon due to the isovector and isoscalar axial-vector currents. For this purpose we start by considering the 
polarization operator, which is the basic object of the sum rule approach,

$$
\Pi_{\mu}(p, q)=i \int d^{4} x e^{i q x}\left\langle 0\left|T\left\{\eta(0) A_{\mu}^{(S)}(x)\right\}\right| N(p)\right\rangle
$$

where

$$
A_{\mu}^{(S)}=\bar{u} \gamma_{\mu} \gamma_{5} u \mp \bar{d} \gamma_{\mu} \gamma_{5} d
$$

with the upper (lower) sign corresponding to the isovector (isoscalar) axial-vector current, and $\eta$ is an interpolating current with nucleon quantum numbers. The nucleon interpolating currents without the derivative terms, and with nucleon quantum numbers, can be written as [10]

$$
\begin{aligned}
& \eta_{1}(x)=2 \epsilon^{a b c} \sum_{\ell=1}^{2}\left(u^{a T}(x) C A_{1}^{\ell} d^{b}(x)\right) A_{2}^{\ell} u^{c}(x), \\
& \eta_{2}(x)=\frac{2}{3} \epsilon^{a b c}\left[\left(u^{a T}(x) C \not u^{b}(x)\right) \gamma_{5} \not d^{c}(x)-\left(u^{a T}(x) C \not d^{b}(x)\right) \gamma_{5} \nless u^{c}(x)\right],
\end{aligned}
$$

where $A_{1}^{1}=I, A_{2}^{1}=\gamma_{5}, A_{1}^{2}=\gamma_{5}, A_{2}^{2}=\beta ; C$ is the charge conjugation operator; $a, b, c$ are the color indices, and $z$ is a light-like vector with $z^{2}=0$. The choice $\beta=-1$ in Eq. (3) corresponds to the Ioffe current [11]. Note that $\eta_{2}$ current is modified in the following way [9]

$$
\eta_{3}(x)=\epsilon^{a b c}\left[\left(u^{a T}(x) C \not u^{b}(x)\right) \gamma_{5} \not d^{c}(x)\right] .
$$

The axial form factor and induced pseudoscalar form factor of the nucleon are calculated in [12 in the framework of LCSR using the current $\eta_{3}(x)$. However the current $\eta_{3}(x)$ couples to both spin $1 / 2$ and $3 / 2$ baryons. Therefore, for a reliable determination of the form factors the unwanted contributions coming from spin $3 / 2$ states should be eliminated. But this elimination is not done in [12].

Note that the form factors $G_{A}\left(Q^{2}\right)$ and $G_{P}\left(Q^{2}\right)$ induced by the isovector axial current are calculated in [13] in LCSR using the Ioffe current.

In the present work we calculate the form factors induced by isovector and isoscalar axial-vector currents using the general form of the interpolating $\eta_{1}(x)$.

We start by calculating the correlation function (11) from the QCD side. At large Euclidean momenta $p^{2}=(p-q)^{2}$ and $q^{2}=-Q^{2}$ the correlation function can be calculated perturbatively. Using the expressions of interpolating currents we get the following result for the correlator:

$$
\begin{aligned}
\Pi_{\mu} & =\frac{1}{2} \int d^{4} x e^{i q x} \sum_{\ell=1}^{2}\left\{\left(C A_{1}^{\ell}\right)_{\alpha \gamma}\left[A_{2}^{\ell} S_{u}(-x) \gamma_{\mu} \gamma_{5}\right]_{\rho \beta} 4 \epsilon^{a b c}\left\langle 0\left|u_{\alpha}^{a}(0) u_{\beta}^{b}(x) d_{\gamma}^{c}(0)\right| N\right\rangle\right. \\
& +\left(A_{2}^{\ell}\right)_{\rho \alpha}\left[\left(C A_{1}^{\ell}\right)^{T} S_{u}(-x) \gamma_{\mu} \gamma_{5}\right]_{\gamma \beta} 4 \epsilon^{a b c}\left\langle 0\left|u_{\alpha}^{a}(x) u_{\beta}^{b}(x) d_{\gamma}^{c}(0)\right| N\right\rangle \\
& \left.\mp\left(A_{2}^{\ell}\right)_{\rho \beta}\left[C A_{1}^{\ell} S_{u}(-x) \gamma_{\mu} \gamma_{5}\right]_{\alpha \gamma} 4 \epsilon^{a b c}\left\langle 0\left|u_{\alpha}^{a}(0) u_{\beta}^{b}(0) d_{\gamma}^{c}(x)\right| N\right\rangle\right\},
\end{aligned}
$$


where $S_{q}(-x)$ is the light quark propagator and its light cone expanded expression is [13]

$$
S(x)=\frac{i \not x}{2 \pi^{2} x^{4}}-\frac{\langle q \bar{q}\rangle}{12}\left(1+\frac{m_{0}^{2} x^{2}}{16}\right)-i g_{s} \int_{0}^{1} d v\left[\frac{\not x}{16 \pi^{2} x^{4}} G_{\mu \nu} \sigma^{\mu \nu}-v x^{\mu} G_{\mu \nu} \gamma^{\nu} \frac{i}{4 \pi^{2} x^{2}}\right] .
$$

The terms proportional to $G_{\mu \nu}$ in Eq. (17) give contribution to four- and five-particle nucleon distribution functions, and these amplitudes are expected to be small [14, 15] which will be neglected in further analysis. Only the first term in Eq. (7) survives since Borel transformation kills the second term.

It follows from Eq. (6) that for the calculation of $\Pi_{\mu}$ we need to know the matrix element $4 \epsilon^{a b c}\left\langle 0\left|u_{\alpha}^{a}\left(a_{1} x\right) u_{\beta}^{b}\left(a_{2} x\right) d_{\gamma}^{c}\left(a_{3} x\right)\right| N(p)\right\rangle$. This matrix element of nonlocal operator is defined in terms of the nucleon distribution amplitudes (DAs), and their explicit expressions are presented in [9, 13, 16, 17, 18].

Using the explicit expressions of the nucleon DAs and performing integration over $x$ and selecting the structures $\phi \gamma_{\mu} \gamma_{5}$ for $G_{A}\left(G_{A}^{S}\right)$ and $q_{\mu} \phi \gamma_{5}$ for $G_{P}\left(G_{P}^{S}\right)$, we get

$$
\begin{aligned}
-\frac{\lambda_{N}}{m_{N}^{2}-p^{2}} G_{A} & =\frac{1}{2}\left\{m_{N} \int_{0}^{1} \frac{d t_{2}}{\left(q-p t_{2}\right)^{2}}\left[(1-\beta) F_{1}\left(t_{2}\right)+(1+\beta) F_{2}\left(t_{2}\right)\right]\right. \\
& \mp \frac{m_{N}}{2} \int_{0}^{1} \frac{d t_{3}}{\left(q-p t_{3}\right)^{2}}\left[2(1-\beta) F_{3}\left(t_{3}\right)+(1+\beta) F_{4}\left(t_{3}\right)\right] \\
& +m_{N}^{3} \int_{0}^{1} \frac{d t_{2}}{\left(q-p t_{2}\right)^{4}}\left[(1-\beta) F_{5}\left(t_{2}\right)+(1+\beta) F_{6}\left(t_{2}\right)\right] \\
& \mp m_{N}^{3} \int_{0}^{1} \frac{d t_{3}}{\left(q-p t_{3}\right)^{4}}\left[(1-\beta) F_{7}\left(t_{3}\right)+(1+\beta) F_{8}\left(t_{3}\right)\right] \\
& +m_{N}^{3} \int_{0}^{1} \frac{d t_{2}}{\left(q-p t_{2}\right)^{4}}\left[(1-\beta) F_{9}\left(t_{2}\right)+(1+\beta) F_{10}\left(t_{2}\right)\right] \\
& \left.\mp m_{N}^{3} \int_{0}^{1} \frac{d t_{3}}{\left(q-p t_{3}\right)^{4}}\left[(1-\beta) F_{11}\left(t_{3}\right)+(1+\beta) F_{12}\left(t_{3}\right)\right]\right\}, \\
\frac{\lambda_{N}}{m_{N}^{2}-p^{2}} G_{P} & =\frac{1}{2}\left\{m_{N}^{2} \int_{0}^{1} \frac{d t_{2}}{\left(q-p t_{2}\right)^{4}}\left[2(1-\beta) F_{13}\left(t_{2}\right)+(1+\beta) F_{14}\left(t_{2}\right)\right]\right. \\
& \left.\mp m_{N}^{2} \int_{0}^{1} \frac{d t_{3}}{\left(q-p t_{3}\right)^{4}}\left[2(1-\beta) F_{15}\left(t_{3}\right)+(1+\beta) F_{16}\left(t_{3}\right)\right]\right\},
\end{aligned}
$$

where upper (lower) sign corresponds to isovector (isoscalar) axial-vector current, and

$$
\begin{aligned}
& F_{1}\left(t_{2}\right)=\int_{0}^{1-t_{2}} d t_{1}\left[-\widetilde{\mathcal{A}}_{2}-2 A_{3}-\widetilde{\mathcal{V}}_{2}+2 V_{1}-2 V_{3}\right]\left(t_{1}, t_{2}, 1-t_{1}-t_{2}\right), \\
& F_{2}\left(t_{2}\right)=\int_{0}^{1-t_{2}} d t_{1}\left[2 P_{1}+2 S_{1}+4 T_{1}-\widetilde{\mathcal{T}}_{2}-8 T_{7}-3 \widetilde{\mathcal{T}}_{4}\right]\left(t_{1}, t_{2}, 1-t_{1}-t_{2}\right), \\
& F_{3}\left(t_{3}\right)=\int_{0}^{1-t_{3}} d t_{1}\left[A_{1}-V_{1}\right]\left(t_{1}, 1-t_{1}-t_{3}, t_{3}\right), \\
& F_{4}\left(t_{3}\right)=\int_{0}^{1-t_{3}} d t_{1}\left[2 P_{1}+2 S_{1}-2 T_{1}+\widetilde{\mathcal{T}}_{2}+4 T_{7}+\widetilde{\mathcal{T}}_{4}\right]\left(t_{1}, 1-t_{1}-t_{3}, t_{3}\right)
\end{aligned}
$$




$$
\begin{aligned}
& F_{5}\left(t_{2}\right)=\int_{0}^{1-t_{2}} d t_{1}\left[2 \widetilde{\mathcal{V}}_{1}^{M}\right]\left(t_{1}, t_{2}, 1-t_{1}-t_{2}\right), \\
& F_{6}\left(t_{2}\right)=\int_{0}^{1-t_{2}} d t_{1}\left[4 \widetilde{\mathcal{T}}_{1}^{M}\right]\left(t_{1}, t_{2}, 1-t_{1}-t_{2}\right), \\
& F_{7}\left(t_{3}\right)=\int_{0}^{1-t_{3}} d t_{1}\left[\widetilde{\mathcal{A}}_{1}^{M}-\widetilde{\mathcal{V}}_{1}^{M}\right]\left(t_{1}, 1-t_{1}-t_{3}, t_{3}\right) \\
& F_{8}\left(t_{3}\right)=\int_{0}^{1-t_{3}} d t_{1}\left[-\widetilde{\mathcal{T}}_{1}^{M}\right]\left(t_{1}, 1-t_{1}-t_{3}, t_{3}\right), \\
& F_{9}\left(t_{2}\right)=\int_{1}^{t_{2}} d \lambda \int_{1}^{\lambda} d \rho \int_{0}^{1-\rho} d t_{1}\left[2 \widetilde{\mathcal{A}}_{6}\right]\left(t_{1}, \rho, 1-t_{1}-\rho\right), \\
& F_{10}\left(t_{2}\right)=\int_{1}^{t_{2}} d \lambda \int_{1}^{\lambda} d \rho \int_{0}^{1-\rho} d t_{1}\left[-2 \widetilde{\mathcal{T}}_{6}+4 \widetilde{\mathcal{T}}_{8}\right]\left(t_{1}, \rho, 1-t_{1}-\rho\right) \\
& F_{11}\left(t_{3}\right)=\int_{1}^{t_{3}} d \lambda \int_{1}^{\lambda} d \rho \int_{0}^{1-\rho} d t_{1}\left[\widetilde{\mathcal{A}}_{6}+\widetilde{\mathcal{V}}_{6}\right]\left(t_{1}, 1-t_{1}-\rho, \rho\right), \\
& F_{12}\left(t_{3}\right)=\int_{1}^{t_{3}} d \lambda \int_{1}^{\lambda} d \rho \int_{0}^{1-\rho} d t_{1}\left[\widetilde{\mathcal{T}}_{6}-\widetilde{\mathcal{T}}_{8}\right]\left(t_{1}, 1-t_{1}-\rho, \rho\right), \\
& F_{13}\left(t_{2}\right)=\int_{1}^{t_{2}} d \rho \int_{0}^{1-\rho} d t_{1}\left[\widetilde{\mathcal{A}}_{2}-2 \widetilde{\mathcal{A}}_{4}+2 \widetilde{\mathcal{A}}_{5}+\widetilde{\mathcal{V}}_{2}-\widetilde{\mathcal{V}}_{5}\right]\left(t_{1}, \rho, 1-t_{1}-\rho\right), \\
& F_{14}\left(t_{2}\right)=\int_{1}^{t_{2}} d \rho \int_{0}^{1-\rho} d t_{1}\left[2\left(\widetilde{\mathcal{P}}_{2}-\widetilde{\mathcal{S}}_{2}\right)+4 \widetilde{\mathcal{T}}_{2}+6 \widetilde{\mathcal{T}}_{4}+10 \widetilde{\mathcal{T}}_{5}-\widetilde{\mathcal{T}}_{6}+20 \widetilde{\mathcal{T}}_{7}\right]\left(t_{1}, \rho, 1-t_{1}-\rho\right), \\
& F_{15}\left(t_{3}\right)=\int_{1}^{t_{3}} d \rho \int_{0}^{1-\rho} d t_{1}\left[-\widetilde{\mathcal{A}}_{2}-\widetilde{\mathcal{A}}_{5}-\widetilde{\mathcal{V}}_{2}+\widetilde{\mathcal{V}}_{5}\right]\left(t_{1}, 1-t_{1}-\rho, \rho\right), \\
& F_{16}\left(t_{3}\right)=\int_{1}^{t_{3}} d \rho \int_{0}^{1-\rho} d t_{1}\left[2\left(\widetilde{\mathcal{P}}_{2}-\widetilde{\mathcal{S}}_{2}\right)+2 \widetilde{\mathcal{T}}_{4}+2 \widetilde{\mathcal{T}}_{5}-\widetilde{\mathcal{T}}_{6}+4 \widetilde{\mathcal{T}}_{7}\right]\left(t_{1}, 1-t_{1}-\rho, \rho\right) .
\end{aligned}
$$

Those functions appearing in Eqs. (8) and (9) are determined as

$$
\begin{aligned}
& \widetilde{\mathcal{V}}_{2}\left(t_{i}\right)=V_{1}\left(t_{i}\right)-V_{2}\left(t_{i}\right)-V_{3}\left(t_{i}\right) \\
& \widetilde{\mathcal{A}}_{2}\left(t_{i}\right)=-A_{1}\left(t_{i}\right)+A_{2}\left(t_{i}\right)-A_{3}\left(t_{i}\right) \\
& \widetilde{\mathcal{A}}_{4}\left(t_{i}\right)=-2 A_{1}\left(t_{i}\right)-A_{3}\left(t_{i}\right)-A_{4}\left(t_{i}\right)+2 A_{5}\left(t_{i}\right) \\
& \widetilde{\mathcal{A}}_{5}\left(t_{i}\right)=A_{3}\left(t_{i}\right)-A_{4}\left(t_{i}\right) \\
& \widetilde{\mathcal{A}}_{6}\left(t_{i}\right)=A_{1}\left(t_{i}\right)-A_{2}\left(t_{i}\right)+A_{3}\left(t_{i}\right)+A_{4}\left(t_{i}\right)-A_{5}\left(t_{i}\right)+A_{6}\left(t_{i}\right) \\
& \widetilde{\mathcal{T}}_{2}\left(t_{i}\right)=T_{1}\left(t_{i}\right)+T_{2}\left(t_{i}\right)-2 T_{3}\left(t_{i}\right) \\
& \widetilde{\mathcal{T}}_{4}\left(t_{i}\right)=T_{1}\left(t_{i}\right)-T_{2}\left(t_{i}\right)-2 T_{7}\left(t_{i}\right) \\
& \widetilde{\mathcal{T}}_{5}\left(t_{i}\right)=-T_{1}\left(t_{i}\right)+T_{5}\left(t_{i}\right)+2 T_{8}\left(t_{i}\right) \\
& \widetilde{\mathcal{T}}_{6}\left(t_{i}\right)=2\left[T_{2}\left(t_{i}\right)-T_{3}\left(t_{i}\right)-T_{4}\left(t_{i}\right)+T_{5}\left(t_{i}\right)+T_{7}\left(t_{i}\right)+T_{8}\left(t_{i}\right)\right] \\
& \widetilde{\mathcal{T}}_{7}\left(t_{i}\right)=T_{7}\left(t_{i}\right)-T_{8}\left(t_{i}\right) \\
& \widetilde{\mathcal{S}}_{2}\left(t_{i}\right)=S_{1}\left(t_{i}\right)-S_{2}\left(t_{i}\right) \\
& \widetilde{\mathcal{P}}_{2}\left(t_{i}\right)=P_{2}\left(t_{i}\right)-P_{1}\left(t_{i}\right)
\end{aligned}
$$

whose explicit expressions are given in [13].

Physical part of the correlation function is obtained by inserting a complete set of states between the currents in Eq. (1) with the same quantum numbers of the current $\eta(x)$. After 
isolating the pole term of the nucleon state, the correlator function (1) can be written as

$$
\Pi_{\mu}=\frac{\left\langle 0|\eta| N\left(p^{\prime}\right)\right\rangle\left\langle N\left(p^{\prime}\right)\left|A_{\mu}^{S}\right| N(p)\right\rangle}{m_{N}^{2}-p^{\prime 2}}+\sum_{h} \frac{\left\langle 0|\eta| h\left(p^{\prime}\right)\right\rangle\left\langle h\left(p^{\prime}\right)\left|A_{\mu}^{S}\right| N(p)\right\rangle}{m_{h}^{2}-p^{\prime 2}}
$$

where $p^{\prime}=p-q$ and $q$ is the momentum carried by the axial-vector current. The second term in (10) describes the higher states and continuum contribution, and $h$ is complete set of the hadrons with the quantum numbers of the ground state nucleon.

Contribution of higher states to the physical part of the sum rules are taken into account using quark-hadron duality, i.e., spectral density for higher states is equal to the perturbative spectral density starting from $s>s_{0}$, where $s_{0}$ is the continuum threshold.

The matrix elements entering to Eq. (10) are defined as

$$
\begin{aligned}
\left\langle 0|\eta| N\left(p^{\prime}\right)\right\rangle & =\lambda_{N} u_{N}\left(p^{\prime}\right) \\
\left\langle N\left(p^{\prime}\right)\left|A_{\mu}^{S}\right| N(p)\right\rangle & =\bar{u}_{N}\left(p^{\prime}\right)\left[\gamma_{\mu} G_{A}^{S}\left(q^{2}\right)+\frac{q_{\mu}}{2 m_{N}} G_{P}^{S}\left(q^{2}\right)\right] \gamma_{5} u_{N}(p) .
\end{aligned}
$$

Substituting Eqs. (11) and (12) into Eq. (10) and selecting the structures $\not \gamma_{\mu} \gamma_{5}$ and $q_{\mu} \not \gamma_{5}$ and performing Borel transformation with respect to the variable $(q-p)^{2}$, which suppresses the continuum and higher state contributions, we get the above-mentioned form factors:

$$
\begin{aligned}
G_{A} & =-\frac{1}{2 \lambda_{N}} e^{m_{N}^{2} / M^{2}}\left\{-m_{N} \int_{x_{0}}^{1} d t_{2} e^{-s\left(t_{2}\right) / M^{2}}\left[(1-\beta) F_{1}\left(t_{2}\right)+(1+\beta) F_{2}\left(t_{2}\right)\right]\right. \\
& \mp\left(-\frac{m_{N}}{2} \int_{0}^{1} d t_{3} e^{-s\left(t_{3}\right) / M^{2}}\left[2(1-\beta) F_{3}\left(t_{3}\right)+(1+\beta) F_{4}\left(t_{3}\right)\right]\right) \\
& +\frac{m_{N}^{3}}{M^{2}} \int_{x_{0}}^{1} \frac{d t_{2}}{t_{2}^{2}} e^{-s\left(t_{2}\right) / M^{2}}\left[(1-\beta) F_{5}\left(t_{2}\right)+(1+\beta) F_{6}\left(t_{2}\right)\right] \\
& +\frac{m_{N}^{3}}{Q^{2}+x_{0}^{2} m_{N}^{2}} e^{-s_{0} / M^{2}}\left[(1-\beta) F_{5}\left(x_{0}\right)+(1+\beta) F_{6}\left(x_{0}\right)\right] \\
& \mp \frac{m_{N}^{3}}{M^{2}} \int_{x_{0}}^{1} \frac{d t_{3}}{t_{3}^{2}} e^{-s\left(t_{3}\right) / M^{2}}\left[(1-\beta) F_{7}\left(t_{3}\right)+(1+\beta) F_{8}\left(t_{3}\right)\right] \\
& \mp \frac{m_{N}^{3}}{Q^{2}+x_{0}^{2} m_{N}^{2}} e^{-s_{0} / M^{2}}\left[(1-\beta) F_{7}\left(x_{0}\right)+(1+\beta) F_{8}\left(x_{0}\right)\right] \\
& +\frac{m_{N}^{3}}{M^{2}} \int_{x_{0}}^{1} \frac{d t_{2}}{t_{2}^{2}} e^{-s\left(t_{2}\right) / M^{2}}\left[(1-\beta) F_{9}\left(t_{2}\right)+(1+\beta) F_{10}\left(t_{2}\right)\right] \\
& +\frac{m_{N}^{3}}{Q^{2}+x_{0}^{2} m_{N}^{2}} e^{-s_{0} / M^{2}}\left[(1-\beta) F_{9}\left(x_{0}\right)+(1+\beta) F_{10}\left(x_{0}\right)\right] \\
& \mp \frac{m_{N}^{3}}{M^{2}} \int_{x_{0}}^{1} \frac{d t_{3}}{t_{3}^{2}} e^{-s\left(t_{3}\right) / M^{2}}\left[(1-\beta) F_{11}\left(t_{3}\right)+(1+\beta) F_{12}\left(t_{3}\right)\right] \\
& \left.\mp \frac{m_{N}^{3}}{Q^{2}+x_{0}^{2} m_{N}^{2}} e^{-s_{0} / M^{2}}\left[(1-\beta) F_{11}\left(x_{0}\right)+(1+\beta) F_{12}\left(x_{0}\right)\right]\right\} \\
G_{P} & =-\frac{1}{2 \lambda_{N}} e^{m_{N}^{2} / M^{2}}\left\{\frac{m_{N}^{2}}{M^{2}} \int_{x_{0}}^{1} d t_{2} e^{-s\left(t_{2}\right) / M^{2}}\left[2(1-\beta) F_{13}\left(t_{2}\right)+(1+\beta) F_{14}\left(t_{2}\right)\right]\right. \\
&
\end{aligned}
$$




$$
\begin{aligned}
& +\frac{m_{N}^{3}}{Q^{2}+x_{0}^{2} m_{N}^{2}} e^{-s_{0} / M^{2}}\left[2(1-\beta) F_{13}\left(x_{0}\right)+(1+\beta) F_{14}\left(x_{0}\right)\right] \\
& \mp \frac{m_{N}^{2}}{M^{2}} \int_{x_{0}}^{1} d t_{3} e^{-s\left(t_{3}\right) / M^{2}}\left[2(1-\beta) F_{15}\left(t_{3}\right)+(1+\beta) F_{16}\left(t_{3}\right)\right] \\
& \left.\mp \frac{m_{N}^{3}}{Q^{2}+x_{0}^{2} m_{N}^{2}} e^{-s_{0} / M^{2}}\left[2(1-\beta) F_{15}\left(x_{0}\right)+(1+\beta) F_{16}\left(x_{0}\right)\right]\right\} .
\end{aligned}
$$

where

$$
\begin{aligned}
\lambda_{N}^{2} & =e^{m_{N}^{2} / M^{2}}\left\{\frac{M^{6}}{256 \pi^{4}} E_{2}(x)\left(5+2 \beta+\beta^{2}\right)-\left(1-\beta^{2}\right) \frac{\langle\bar{u} u\rangle}{6}[6\langle\bar{d} d\rangle+\langle\bar{u} u\rangle]\right. \\
& \left.+\left(1-\beta^{2}\right) \frac{m_{0}^{2}}{24 M^{2}}\langle\bar{u} u\rangle[12\langle\bar{d} d\rangle+\langle\bar{u} u\rangle]\right\},
\end{aligned}
$$

and

$$
E_{2}\left(s_{0} / M^{2}\right)=1-e^{s_{0} / M^{2}} \sum_{k=0}^{2} \frac{\left(s_{0} / M^{2}\right)^{k}}{k !} .
$$

In performing Borel transformation, we use the following substitution rules (see for example [12, 13] and [19])

$$
\begin{aligned}
& \int d x \frac{\rho(x)}{(q-x p)^{2}} \rightarrow-\int \frac{d x}{x} \rho(x) e^{-s / M^{2}} \\
& \int d x \frac{\rho(x)}{(q-x p)^{4}} \rightarrow \frac{1}{M^{2}} \int \frac{d x}{x^{2}} \rho(x) e^{-s / M^{2}}+\frac{\rho\left(x_{0}\right)}{Q^{2}+x_{0}^{2} m_{N}^{2}} e^{-s_{0} / M^{2}}
\end{aligned}
$$

where

$$
s(x)=(1-x) m_{N}^{2}+\frac{1-x}{x} Q^{2},
$$

and $x_{0}$ is the solution of the quadratic equation for $s=s_{0}$, i.e.,

$$
x_{0}=\frac{1}{2 m_{N}^{2}}\left[\sqrt{\left(Q^{2}+s_{0}-m_{N}^{2}\right)^{2}+4 m_{N}^{2} Q^{2}}-\left(Q^{2}+s_{0}-m_{N}^{2}\right)\right],
$$

and $Q^{2}=-q^{2}$

\section{Results and discussion}

Now we are ready to examine the sum rules for the form factors. It follows from expressions of the sum rules for form factors that DAs are the main input parameters of LCSR. The complete list of all DAs which enter to the sum rules for form factors can be found in [13.

These DAs contain eight hadronic parameters $f_{N}, \lambda_{1}, \lambda_{2}, V_{1}^{d}, A_{1}^{u}, f_{1}^{d}, f_{2}^{d}$ and $f_{1}^{u}$ which should be determined in the framework of various models. In further numerical calculations we consider three different sets of these parameters: 
- QCD sum rules based DAs, in which the parameters are determined from QCD sum rules (set 1 ) having the values $A_{1}^{u}=0.38 \mp 0.15, V_{1}^{d}=0.23 \mp 0.03, f_{1}^{d}=0.40 \mp 0.05$, $f_{2}^{d}=0.22 \mp 0.05, f_{1}^{u}=0.07 \mp 0.05$.

- A model for nucleon DAs (set 2) in which the above-mentioned parameters are chosen in such a way that the form of nucleon DAs describe well the existing experimental data on nucleon form factors (see [13]), whose numerical values are $A_{1}^{u}=1 / 4, V_{1}^{d}=$ $13 / 42$ and the values of $f_{1}^{d}, f_{2}^{d}$ and $f_{1}^{u}$ are the same as in set 1 .

- Asymptotic forms of DAs of all twists (set 3) in which the values of the parameters are set to $A_{1}^{u}=0, V_{1}^{d}=1 / 3, f_{1}^{d}=3 / 10, f_{2}^{d}=4 / 15, f_{1}^{u}=1 / 10$.

Note that the values of $f_{N}, \lambda_{1}$ and $\lambda_{2}$ for these three sets are the same, i.e., $f_{N}=$ $(5.0 \mp 0.5) \times 10^{-3} \mathrm{GeV}^{2}, \lambda_{1}=-(2.7 \mp 0.9) \times 10^{-2} \mathrm{GeV}^{2}$ and $\lambda_{2}=(5.4 \mp 1.9) \times 10^{-2} \mathrm{GeV}^{2}$.

The values of the non-perturbative parameters entering to DAs at $\mu=1 \mathrm{GeV}$ scale are given in [9] and [13]. Sum rules for the form factors involve three unphysical parameters, namely, continuum threshold $s_{0}$, Borel parameter $M^{2}$ and the parameter $\beta$ in the interpolating current of nucleon. Of course, if we can perform OPE up to infinite order, the result must be independent of these parameters. But we truncated OPE in the finite order, and hence, there appears dependence of sum rules on these auxiliary parameters. However, any physical quantity can not depend on unphysical parameters. Therefore, in the first hand, we should find the appropriate regions of unphysical parameters where form factors are independent on them. From an analysis of mass sum rules it follows that, when continuum threshold $s_{0}$ lies in the region $2 \mathrm{GeV}^{2} \leq s_{0} \leq 2.5 \mathrm{GeV}^{2}$, the prediction of sum rules on the mass of the baryons are practically independent of $s_{0}$. It is this region of $s_{0}$ which we will use in our numerical calculations.

Having determined $s_{0}$, we next try to find a region (the so called working region) of $M^{2}$ where the above-mentioned form factors are independent of $M^{2}$ at fixed values of $s_{0}$ and $\beta$. We study the dependence of the form factors induced by the axial-vector current on $M^{2}$ at fixed values of $Q^{2}$ and $\beta$ at $s_{0}=2.0 \mathrm{GeV}^{2}$ and $s_{0}=2.5 \mathrm{GeV}^{2}$, for three different sets of the wave functions. We obtain that the results are almost the same for both choices of $s_{0}$.

An upper bound for the Borel parameter $M^{2}$ is determined by requiring that the contribution of continuum be less compared to the continuum subtracted sum rules. Lower limit is determined from the condition that the contribution of the term with highest power of $1 / M^{2}$ is less, say $30 \%$, compared to the higher powers of $M^{2}$ term contribution. Using these constraints we found that the working region of $M^{2}$ is $1 \mathrm{GeV}^{2} \leq M^{2} \leq 2.5 \mathrm{GeV}^{2}$, and we obtain that the results are rather stable with respect to the variations of $M^{2}$, when $M^{2}$ lies in the above-mentioned working region. It is also observed that the results of for the form factors depend also on the parameter $\beta$. But, as has already been mentioned, the parameter $\beta$ is an auxiliary quantity and therefore the form factors must be independent of it. As a result of this argument, we need to find a region for $\beta$, where the results for the form factors are independent of its value. Analyses of mass sum rules [19] and meson-octet baryon couplings [20] lead to the result $\beta<-1.3$ and $\beta>3.3$. In our numerical calculations we will use these bounds for $\beta$. It should be noted here that the Monte Carlo analysis of mass sum rules for baryons [21] predicts an optimal value $\beta \simeq-1.2$, which is close to our choice of lower bound. 
The correlation functions can be calculated in QCD for sufficiently large negative values of $Q^{2}$ and $(p-q)^{2}$ using OPE. The form factors can reliably be determined at the range $Q^{2} \geq 2 \mathrm{GeV}^{2}$. Our approach is not applicable for smaller values of $Q^{2}$. For this reason, the form factors are evaluated in the range $Q^{2} \geq 2 \mathrm{GeV}^{2}$. We study the dependence of the form factors on $Q^{2}$ at fixed values of $\beta$, lying within the above-mentioned working region, at fixed values of $M^{2}=2 \mathrm{GeV}^{2}$ and $s_{0}=2.25 \mathrm{GeV}^{2}$, for the three sets of DAs.

From numerical analysis we obtain the following results:

- The form factors $G_{A}\left(Q^{2}\right)$ and $G_{P}\left(Q^{2}\right)$ exhibit practically the same $Q^{2}$ dependence for all three sets of the nucleon DAs.

- The values of the form factor $G_{A}\left(Q^{2}\right)$ almost coincide at negative values of $\beta$ for the second and third set of DAs, while it differs about $50 \%$ at $\beta=-1.4$ and $15 \%$ at $\beta=-5$, for the first set of DAs. For the positive values of $\beta, G_{A}\left(Q^{2}\right)$ practically coincide for all three sets of DAs.

- The values of $G_{P}\left(Q^{2}\right)$ are very close to each other for all three sets of DAs.

- The values of $G_{A}^{S}\left(Q^{2}\right)$ are very close to each other for the second and third sets of DAs, but it is larger about $50 \%$ for the first set of DAs at $\beta=-1.4$.

- The difference between the values of $G_{P}^{S}\left(Q^{2}\right)$ is very small for all three sets.

- We see that all form factors are negative (positive) at positive (negative) values of $\beta$.

For illustration, in Figs. (1)-(4) we present the dependencies of the form factors $G_{A}\left(Q^{2}\right)$, $G_{P}\left(Q^{2}\right), G_{A}^{S}\left(Q^{2}\right)$ and $G_{P}^{S}\left(Q^{2}\right)$ on $Q^{2}$ at several fixed values of $\beta$, including the results for $\beta=-1$ case.

As has already been noted, the axial form factor $G_{A}\left(Q^{2}\right)$ is considered in [13], however the analytical results that are presented in that work and in ours should be different, since the considered structures are different. However, we see that our numerical results on $G_{A}\left(Q^{2}\right)$ are close to that obtained in [13] at $\beta=-1$.

Note that for the analysis of the existing data from neutrino scattering experiments, form factor $G_{A}\left(Q^{2}\right)$ is usually parametrized in the dipole form

$$
G_{A}^{(d)}\left(Q^{2}\right)=\frac{g_{A}}{\left(1+Q^{2} / m_{A}^{2}\right)^{2}}
$$

where $g_{A}=1.2695 \mp 0.0029$, which is determined from $\beta$ decay [2]. The global average for $m_{A}$ extracted from neutrino scattering experiment is predicted to have the value $m_{A}=$ $(1.026 \mp 0.021) \mathrm{GeV}$ in [22], while the value $m_{A}=(1.20 \mp 0.12) \mathrm{GeV}$ announced by the $\mathrm{K} 2 \mathrm{~K}$ collaboration is slightly larger [23].

In Figs. (5)-(10) we present the LCSR prediction for the axial form factor $G_{A}\left(Q^{2}\right)$ normalized to $G_{A}^{(d)}\left(Q^{2}\right)$, for all three sets of DAs. Experimentally this ratio, i.e., $G_{A}\left(Q^{2}\right) / G_{A}^{(d)}\left(Q^{2}\right)$ should be close to 1 . From these figures we see that the prediction for this ratio by LCSR is quite close to 1 at $\beta=-1.4$ for the first (third) set of DAs and for the choice of the mass $m_{A}=1.0 \mathrm{GeV}\left(m_{A}=1.2 \mathrm{GeV}\right)$. The ratio $R\left(Q^{2}\right)=G_{A}\left(Q^{2}\right) / G_{A}^{d}\left(Q^{2}\right)$ is close to 1 for the second set of DAs at $\beta=-1$ for the choice of the mass $m_{A}=1.2 \mathrm{GeV}$. But, as 
has already been noted, $\beta$ should be different from -1 . Therefore we can conclude that the dipole form of the $G_{A}\left(Q^{2}\right)$ describes well only the first set of DAs at $\beta=-1$. Our results on $G_{A}\left(Q^{2}\right)$ are in close agreement with the chiral quark model [24] predictions and lattice results [25] at $\beta=-1.4$. The results for $G_{A}\left(Q^{2}\right)$ for other values of $\beta$ depart considerably from the lattice results.

It should be remembered that all available experimental data exists only at low $Q^{2}$ region. But unfortunately, our approach can give reliable prediction about the form factors only at high $Q^{2} \geq 2 \mathrm{GeV}^{2}$ region. Therefore direct comparison of our theoretical prediction on experimental result is impossible. Recently proposed experiment Minerva [26] would provide a precise determination of $G_{A}$ at $Q^{2}<2 \mathrm{GeV}^{2}$ and $Q^{2}>2 \mathrm{GeV}^{2}$. Another experiment using $e+p \rightarrow \nu+n$ has been planned at JLAB [27, covering the range $Q^{2}=1 \div 3 \mathrm{GeV}^{2}$. When the result of these experiments will become available, it will be possible to compare the theoretical and experimental results. As the final remark we would like to note that, for a more reliable prediction of the form factors, it is necessary to take into account the radiative $\alpha_{s}$ corrections and the distribution amplitudes with four, five particles.

In conclusion, in this work we calculate the form factors of nucleons induced by isovector and isoscalar axial-vector currents in LCSR method using the general form of the nucleon interpolating current. In our calculations we use three different sets. The dependence of the form factor $G_{A}\left(Q^{2}\right)$ on $Q^{2}$ is compared with its dipole form parametrization which follows from the analysis of neutrino experiments. Our analysis show the results for $G_{A}\left(Q^{2}\right)$ in LCSR, for the first set of DAs at $\beta=-1.4$, well describes the dipole form of $G_{A}\left(Q^{2}\right)$.

\section{Acknowledgments}

One of the authors (T. M. A) is grateful to TÜBİTAK for partially support of this work under the project $105 \mathrm{~T} 131$. 


\section{References}

[1] M. R. Schindler and S. Scherer, prep: hep-ph/0608325 (2006).

[2] W. -M. Yao et al., Particle Data J. Phys. G 33, 1 (2006).

[3] I. I. Balitsky, V. M. Braun, A. V. Kolesnichenko, Nucl. Phys. B 312, 509 (1989); V. L. Chernyak and A. R. Zhitnitsky, Phys. Rept. 112, 173 (1984).

[4] V. M. Braun, prep: hep-ph/9801222 (1998); P. Colangelo, A. Khodjamirian, in " At the Frontier of Particle Physics/Handbook of QCD" ed. by M. Shifman (Worl Scientific, Singapore, 2001), Volume 3, 1495.

[5] M. A. Shifman, V. I. Vainshtein, V. I. Zakharov, Nucl. Phys. B 147, 385 (1979).

[6] V. M. Braun, A. Lenz, N. Mahnke, E. Stein, Phys. Rev. D 65, 074011 (2002); A. Lenz, M. Wittmann and E. Stein, Phys. Lett. B 581, 199 (2004).

[7] Zhi-Gang Wang, Shao-Long Wan and Wei-Min Yang, Phys. Rev. D 73, 094011 (2006); T. Aliev, M. Savc1, Phys. Rev. D 75, 045006 (2007).

[8] M. Q. Huang, D. W. Wang, Phys. Rev. D 69, 094003 (2004).

[9] V. M. Braun, R.J. Fries, N. Mahnke, E. Stein, Nucl. Phys. B 589, 381 (2000), Erratumibid. B 607, 433 (2001).

[10] B. L. Ioffe, Z. Phys. C 18, 67 (1983); Y. Chang, H. G. Dosh, M. Kremer and D. Schall, Nucl. Phys. B 197, 55 (1982).

[11] B. L. Ioffe, Nucl. Phys. B 188, 317 (1981); Erratum-ibid, B 191, 591 (1981).

[12] Zhi-Gang Wang, Shao-Long Wan and Wei-Min Yang, Eur. Phys. J. C 47, 375 (2006).

[13] V. M. Braun, A. Lenz, M. Wittmann, Phys. Rev. D 73, 094019 (2006).

[14] I. I. Balitsky and V. M. Braun, Nucl. Phys. B 311, 541 (1989);

[15] M. Diehl, T. Feldmann, R. Jakob and P. Kroll, Eur. Phys. J. C 8, 409 (1999).

[16] V. M. Braun, A. Lenz, G. Peters, A. V. Radyushkin, Phys. Rev. D 73, 034020 (2006).

[17] V. L. Chernyak and A. R. Zhitnitsky, Nucl. Phys. B 246, 52 (1984).

[18] G. P. Lepage and S. Brodsky, Phys. Rev. Lett. 43, 595 (1979); S. J. Brodsky, G. P. Lepage and A. A. Zaidi, Phys. Rev. D 23, 1152 (1981).

[19] T. M. Aliev, A. Özpineci, M. Savcı, Phys. Rev. D 66, 016002 (2002).

[20] T. M. Aliev, A. Özpineci, S. Yakovlev and V. Zamiralov, Phys. Rev. D 74, 116001 (2006).

[21] D. B. Leinweber, Ann. Phys. 254, 328 (1997). 
[22] V. Bernard, L. Elouadrhiri and U. G. Meißner, J. Phys. G 28, R1 (2002).

[23] R. Gran et al., K2K Collaboration, Phys. Rev. D 74, 052002 (2006).

[24] A. Silva, H. C. Kim, D. Urbano, K. Goeke, Phys. Rev. D 72, 094011 (2005).

[25] M. Göckeler et al., prep: hep-lat/0609001 (2006).

[26] K. McFarland, Minerva Proposal.

[27] A. Deur, Jefferson Laboratory, PAC25, Letter of Intend LOI-04-006. 


\section{Figure captions}

Fig. (1) The dependence of the nucleon form factor $G_{A}\left(Q^{2}\right)$ on $Q^{2}$ at $M^{2}=2 G e V^{2}$ and $s_{0}=2.25 \mathrm{GeV}^{2}$, at four different values of $\beta$ : $\beta=-5, \beta=-1.4 \beta=-1$ and $\beta=5$, for the first set of DAs.

Fig. (2) The same as in Fig. (1), but for the form factor $G_{P}\left(Q^{2}\right)$.

Fig. (3) The same as in Fig. (1), but for the form factor $G_{A}^{S}\left(Q^{2}\right)$.

Fig. (4) The same as in Fig. (1), but for the form factor $G_{P}^{S}\left(Q^{2}\right)$.

Fig. (5) The dependence of the ratio $R=G_{A}\left(Q^{2}\right) / G_{A}^{d}\left(Q^{2}\right)$ on $Q^{2}$ at three fixed values of $\beta$, for the first set of DAs at $m_{A}=1.0 \mathrm{GeV}$.

Fig. (6) The same as in Fig. (5), but for $m_{A}=1.2 \mathrm{GeV}$.

Fig. (7) The same as in Fig. (5), but for the second set of DAs.

Fig. (8) The same as in Fig. (6), but for the second set of DAs.

Fig. (9) The same as in Fig. (5), but for the third set of DAs.

Fig. (10) The same as in Fig. (6), but for the third set of DAs. 


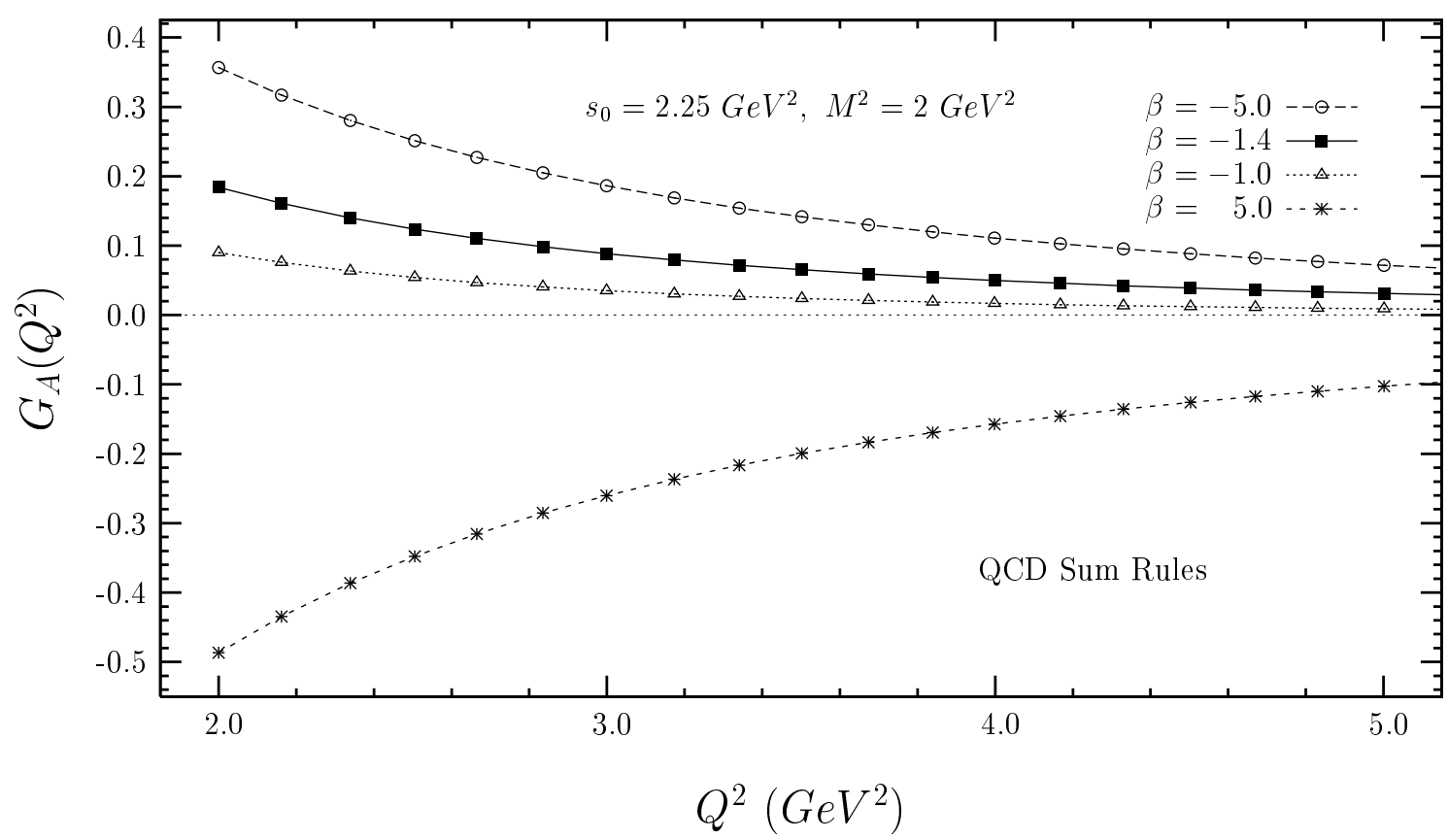

Figure 1:

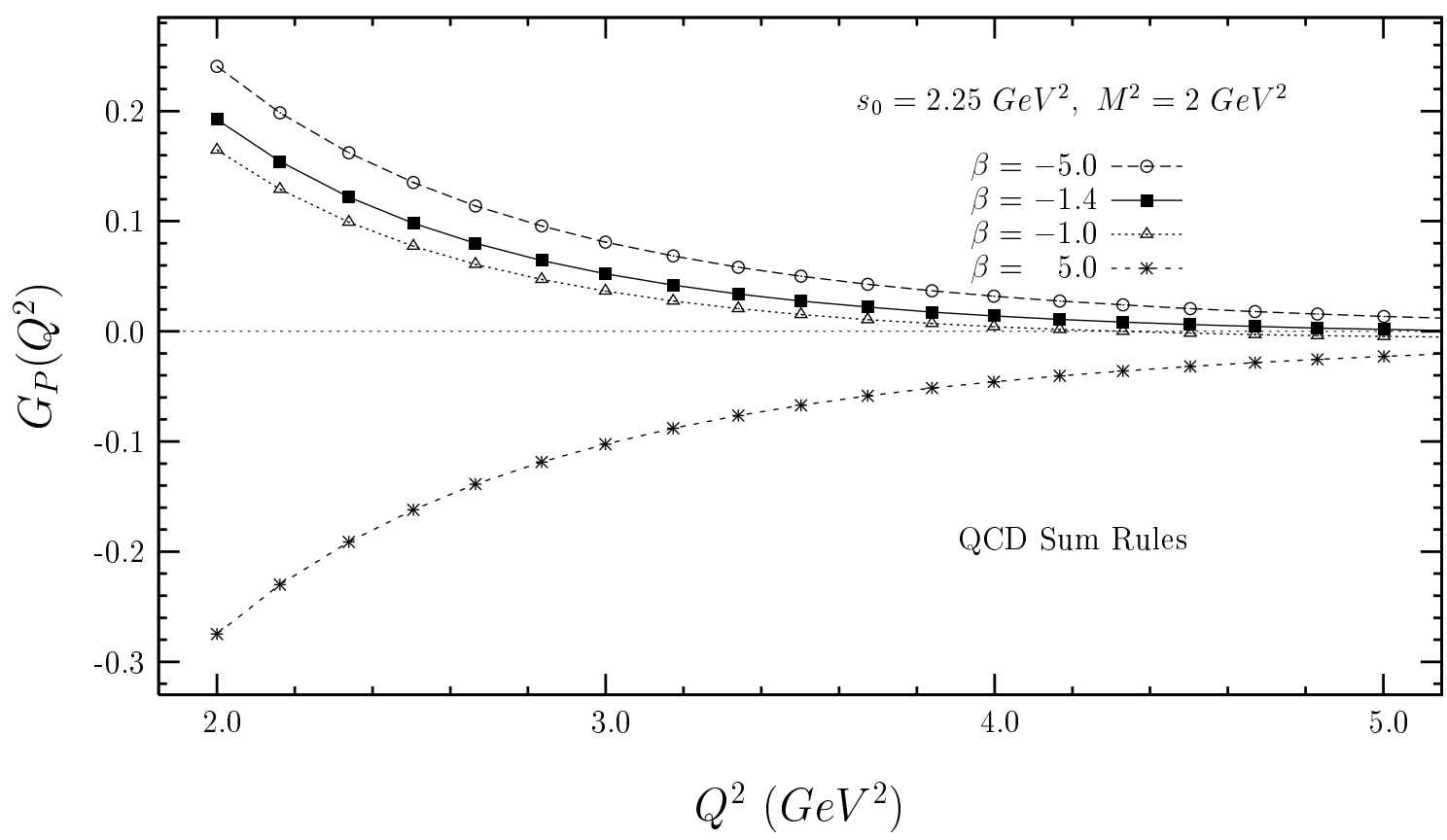

Figure 2: 


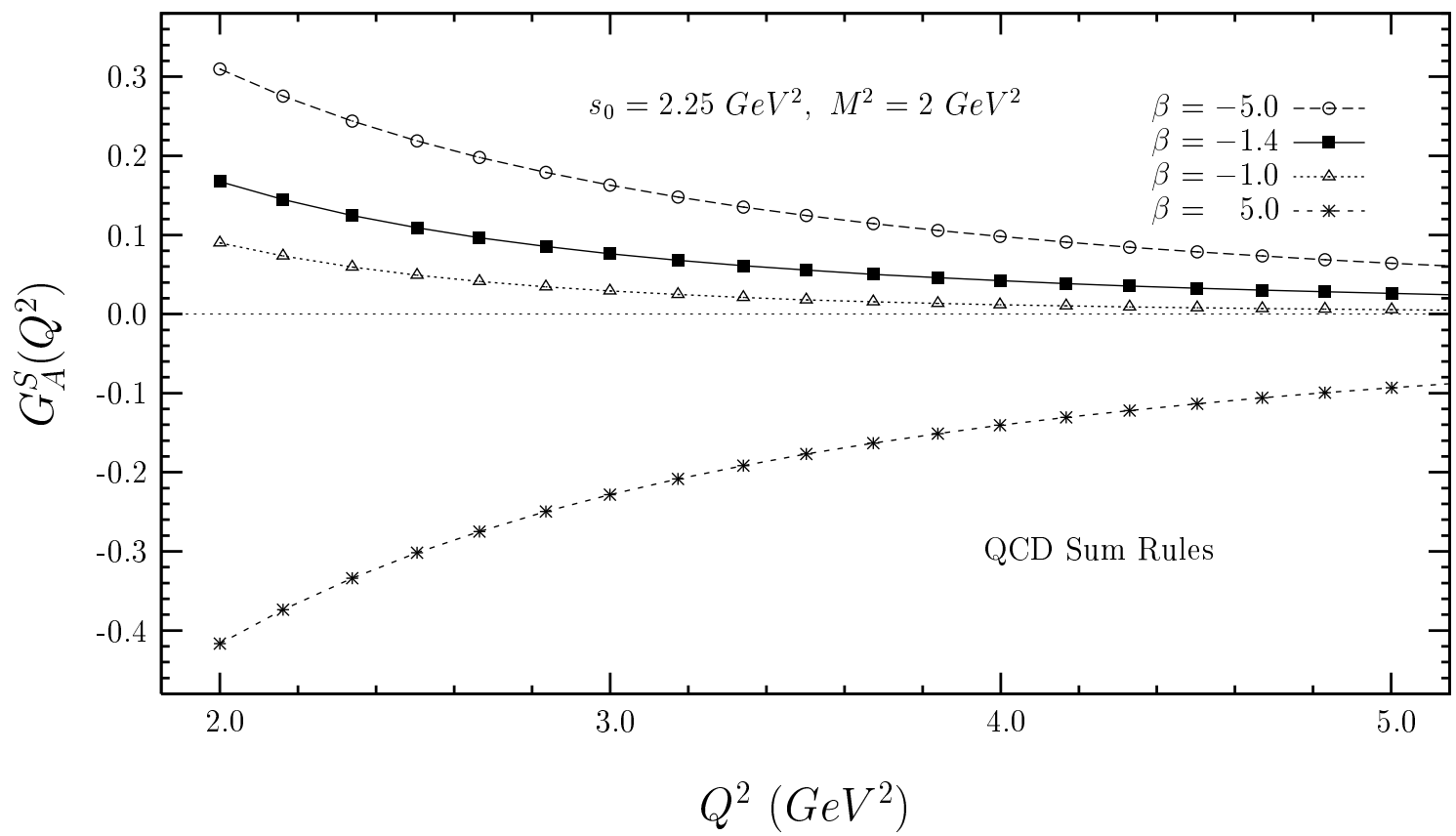

Figure 3:

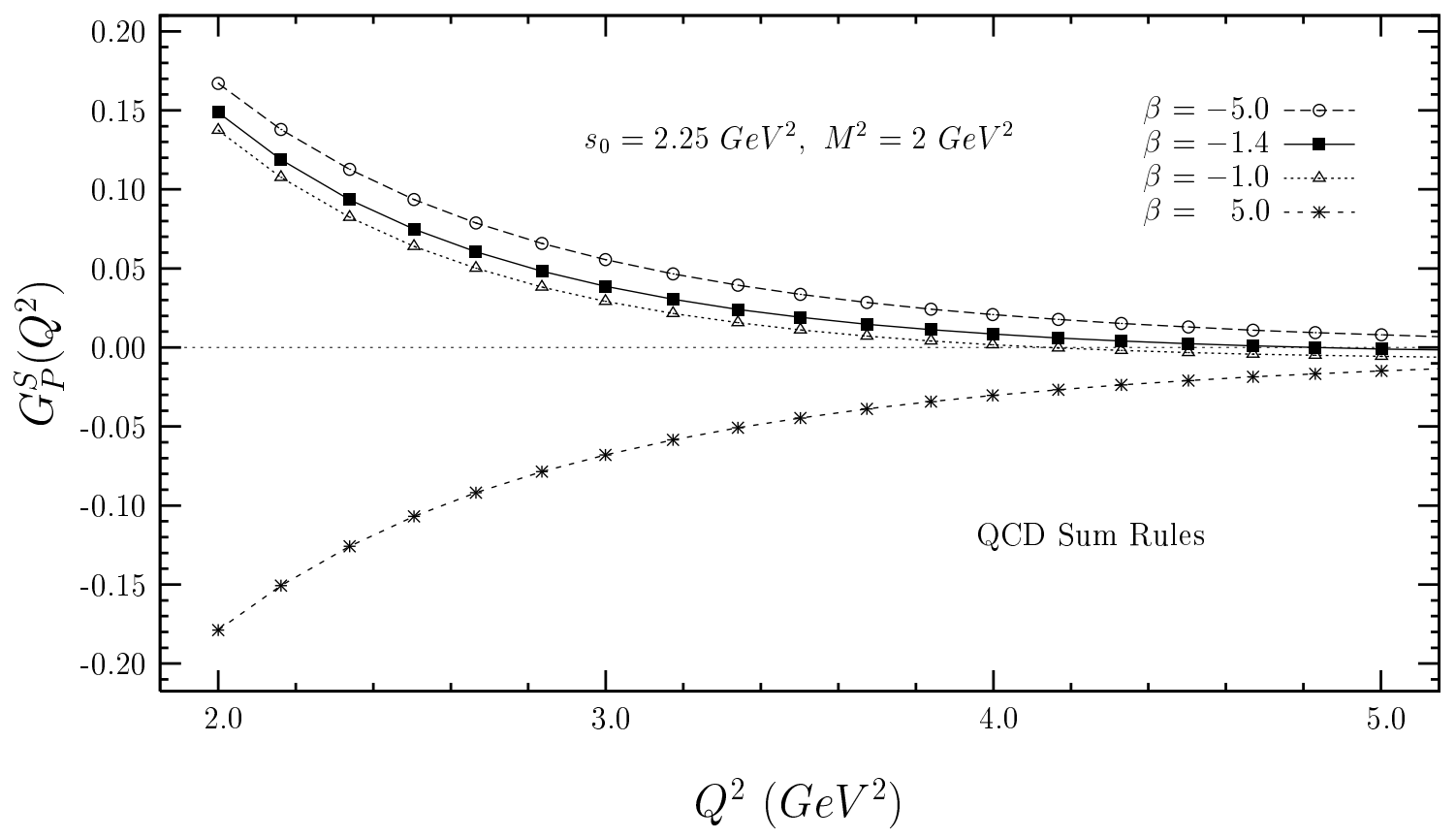

Figure 4: 


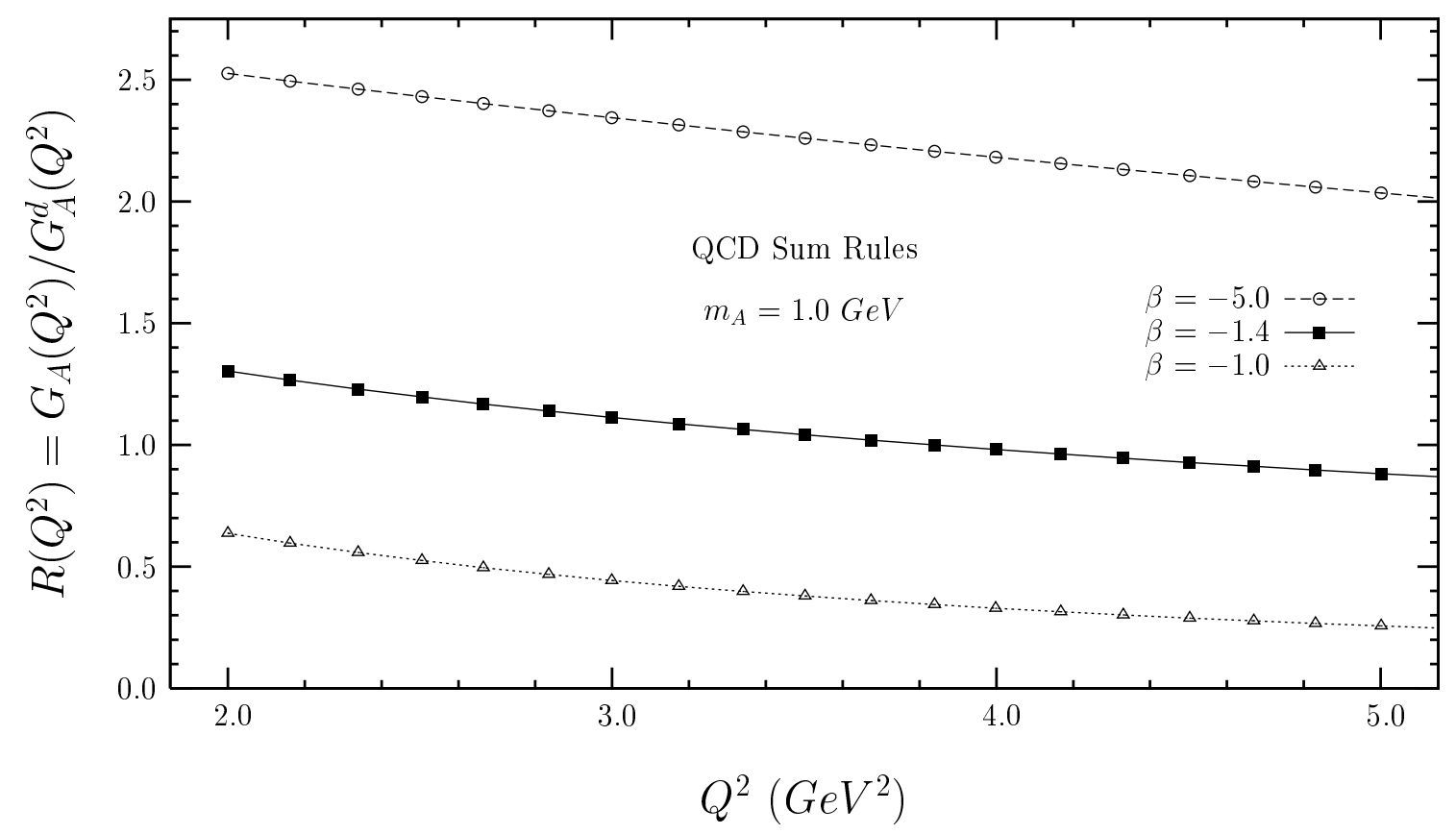

Figure 5:

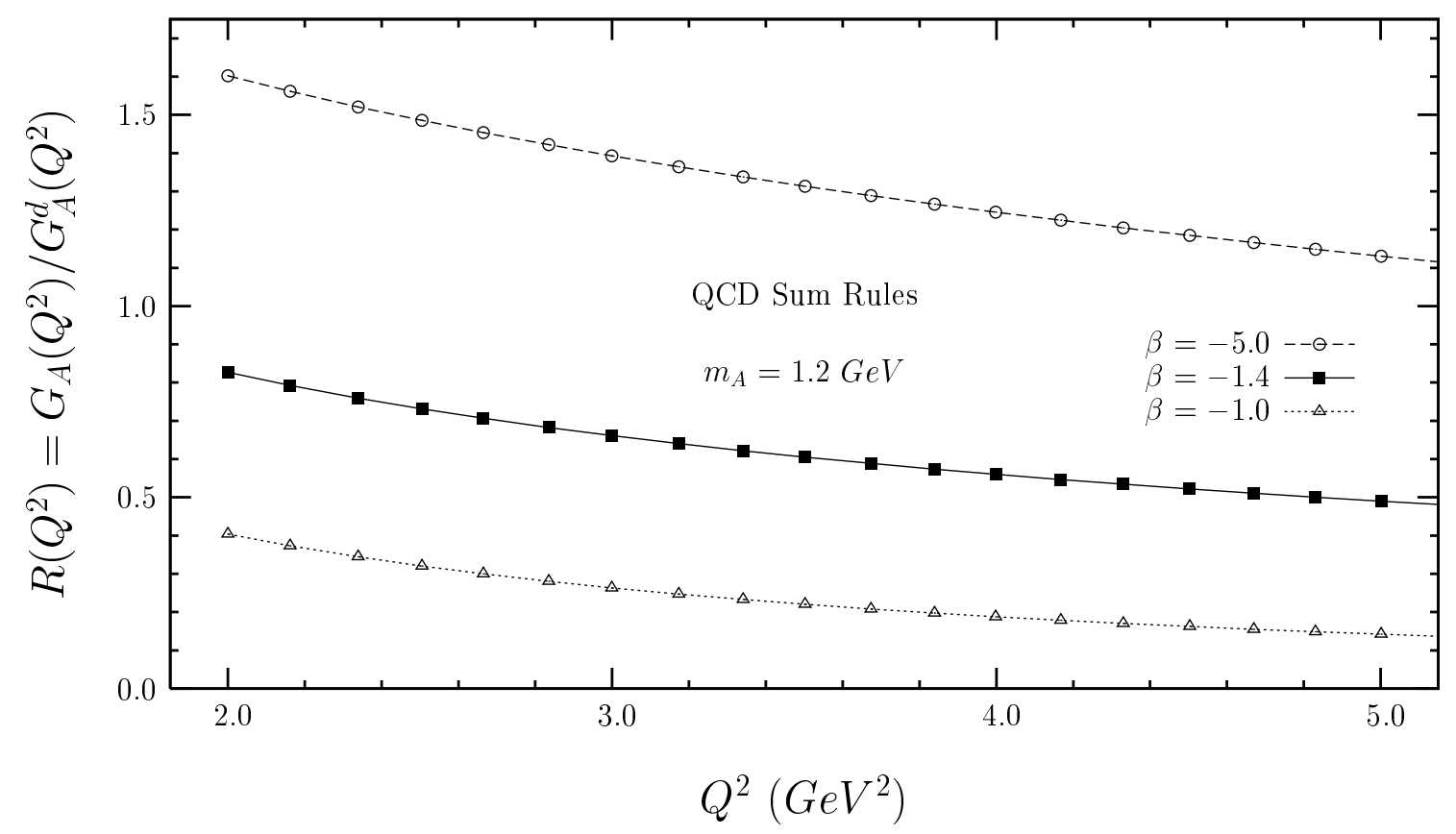

Figure 6: 


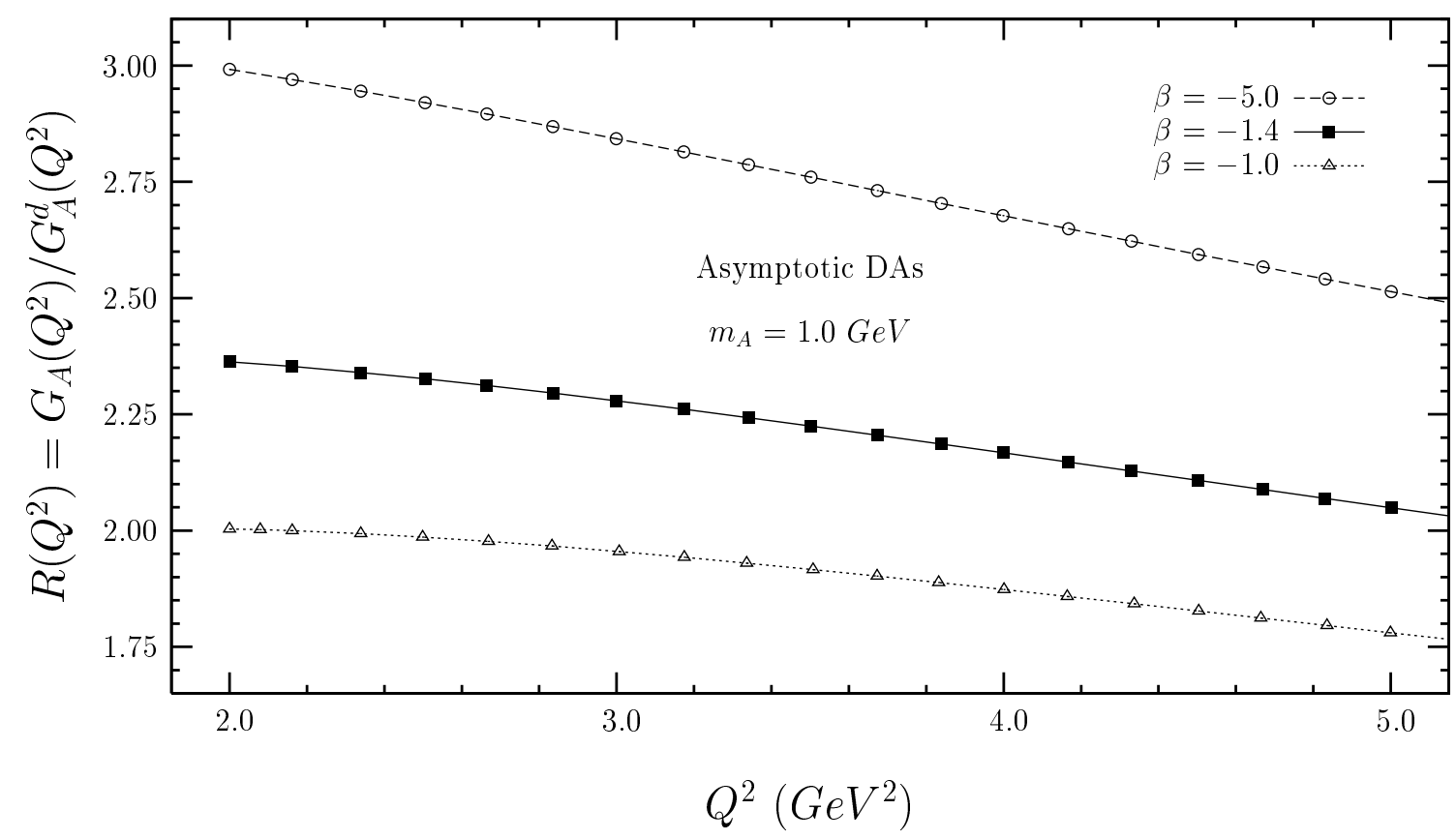

Figure 7:

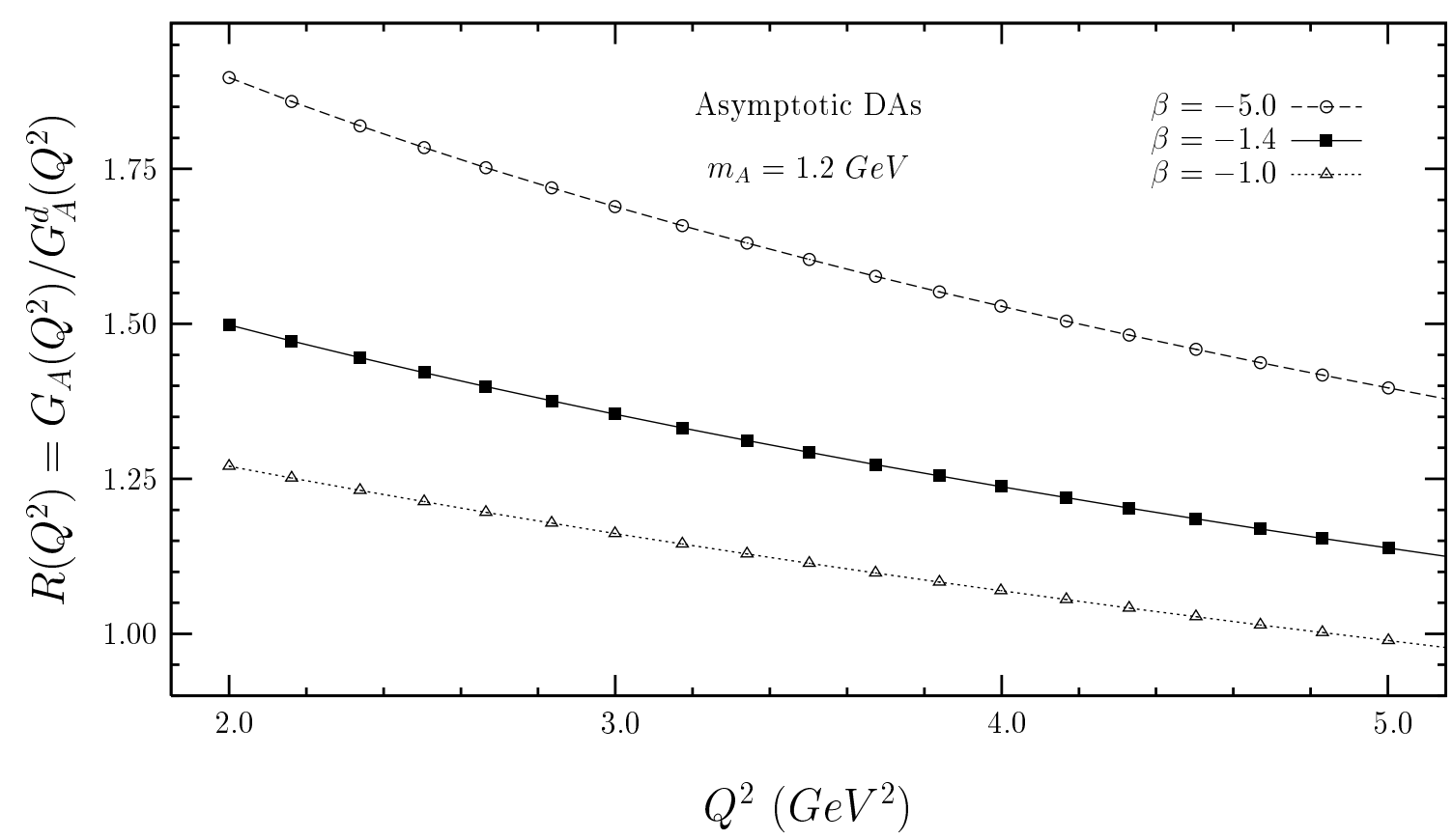

Figure 8: 


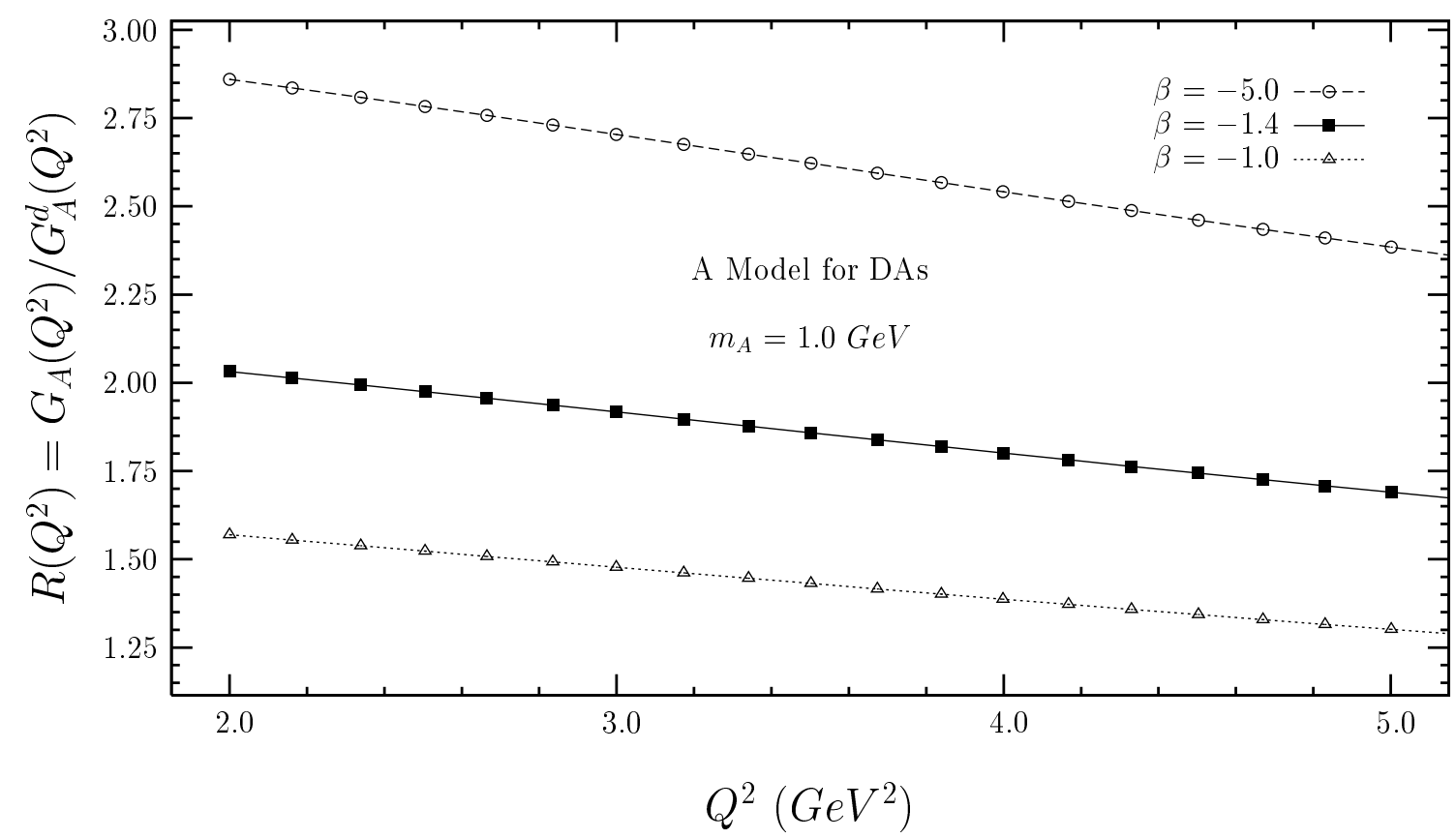

Figure 9:

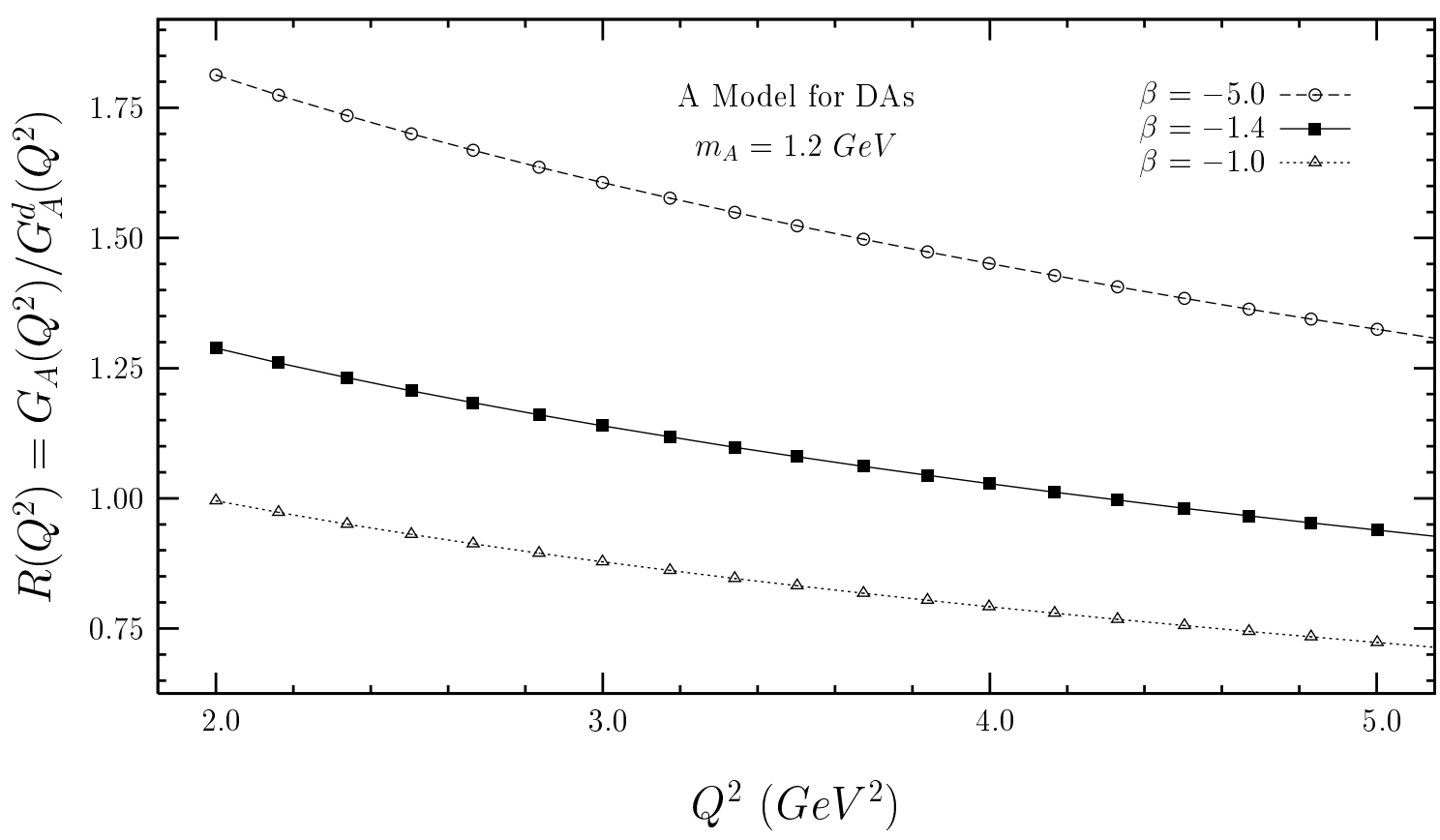

Figure 10: 